\title{
SIADH secundario a adenocarcinoma seroso de ovario tratado con Tolvaptán
}

\author{
Marta Araujo Castro ${ }^{1}$, Ainhoa Abad López ${ }^{1}$, Lourdes Gutiérrez Sanz ${ }^{2}$, Nuria Palacios \\ García 1.
}

Servicio de Endocrinología y Nutrición ${ }^{1}$, Servicio de Oncología Médica ${ }^{2}$. Hospital Puerta de Hierro de Majadahonda, Madrid, España.

\section{RESUMEN}

La hiponatremia es la alteración electrolítica más frecuente en el medio hospitalario, y en un $30 \%$ de los casos se debe a un síndrome de secreción inapropiada de vasopresina (SIADH). EI SIADH está descrito como cuadro paraneoplásico endocrinológico en múltiples tumores, entre los que excepcionalmente se encuentra el de ovario y las neoplasias ginecológicas en general. Presentamos un caso de SIADH paraneoplásico por un citoadenocarcinoma seroso de ovario de alto grado, estadio IV. Se trata del primer caso de SIADH crónico por cáncer de ovario tratado con Tolvaptán. En el presente caso el objetivo de eunatremia se alcanzó con una dosis baja de acuarético, lo que apoya la elevada sensibilidad, ya previamente documentada, de los SIADH tumorales al tratamiento con Tolvaptán.

\section{PALABRAS CLAVE: Síndrome de secreción inapropiada de ADH (SIADH), hiponatremia, tolvaptán}

\section{SUMMARY}

Hyponatremia is the most common electrolyte disturbance in hospitals, and $30 \%$ of cases are due to syndrome of inappropriate secretion of antidiuretic hormone (SIADH). SIADH is described as an endocrine paraneoplastic syndrome in multiple tumors including, ovary and gynecological malignancies in general, although these are exceptional. We report a case of paraneoplastic SIADH for high-grade serous ovarian cystoadenocarcinoma stage IV. This is the first case of chronic SIADH for ovarian cancer treated with Tolvaptan. In this case the target of eunatremia was reached with a low dose of aquaretic, which supports the high sensitivity, as previously documented, of paraneoplasic SIADH to Tolvaptan.

\section{KEY WORDS: Syndrome of inappropriate secretion of antidiuretic hormone (SIADH), hyponatremia, tolvaptán}

\section{INTRODUCCIÓN}

La hiponatremia es un trastorno electrolítico definido como una concentración de sodio en sangre por debajo de $135 \mathrm{mmol} / \mathrm{l}$. Se considera la alteración electrolítica más frecuente en el medio hospitalario, y en un $30 \%$ de los casos se debe a un síndrome de secreción inapropiada de vasopresina (SIADH) (1). EI SIADH a su vez puede ser debido a múltiples causas, entre las que destaca la etiología tumoral. EI SIADH está descrito como cuadro paraneoplásico endocrinológico en 
múltiples tumores, entre los que excepcionalmente se encuentra el de ovario, y las neoplasias ginecológicas en general (2). Los síntomas de la hiponatremia pueden confundirse con frecuencia con la clínica causada por el propio tumor, sobretodo en caso de hiponatremias crónicas.

El tratamiento ideal en los SIADH tumorales es el control adecuado de la neoplasia subyacente, que como ocurre con los adenocarcinomas de ovario, con frecuencia no es posible por la presencia de diseminación en el momento del diagnóstico, lo que hace necesario el plantearse otras medidas terapeúticas para controlar la hiponatremia. Entre estas destaca el Tolvaptán, fármaco muy eficaz en el SIADH, disponible para su administración oral y con escasos efectos secundarios (3). Además es destacable la elevada sensibilidad de los SIADH tumorales a los acuaréticos.

Presentamos el caso de una paciente con un SIADH tumoral por un adenocarcinoma seroso de ovario que presentó una excelente respuesta al tratamiento con dosis bajas de Tolvaptán.

\section{Caso clínico}

Mujer de 42 años remitida a consultas de Endocrinología en Diciembre del 2014 para estudio y tratamiento de hiponatremia. En junio de 2013 había sido diagnosticada de cistoadenocarcinoma seroso de ovario de alto grado, estadío IV (metástasis ganglionares y peritoneales). Inicialmente se trató con cirugía radical y posteriormente, entre julio y noviembre del 2013 recibió 7 sesiones de quimioterapia (QMT) adyuvante con carboplatino + paclitaxel. En octubre de 2014 se evidenció progresión peritoneal y aparición de metástasis hepáticas. Al revisar la historia clínica se objetivó hiponatremia entre 125-134 mmol/l (normal: $135-145 \mathrm{mmol} / \mathrm{l}$ ) desde julio de 2013, con valores previos normales. En la anamnesis dirigida la paciente negaba toma de diuréticos y no refería clínica gastrointestinal ni neurológica. No presentaba edemas y el resto de la exploración física era normal. Con la impresión clínica de hiponatremia euvolémica se procedió a su estudio (Tabla I), confirmándose el diagnóstico de SIADH.

Las pruebas de imagen descartaron patología pulmonar y del SNC por lo que finalmente se estableció el diagnóstico de SIADH crónico de origen paraneoplásico por cistoadenocarcinoma seroso de ovario.

La paciente ingresó en Endocrinología para inicio de tratamiento con Tolvaptán. Tras la administración de una primera dosis de $15 \mathrm{mg}$ presentó una marcada elevación de la natremia (mayor de 6 $\mathrm{mmol} / \mathrm{l}$ en 6 horas) por lo que al alta se indicó una dosis de mantenimiento de $15 \mathrm{mg} / 72 \mathrm{~h}$.

En enero de 2015 ingresó para rescate quirúrgico (resección de adenopatías paraaórticas y metástasis hepáticas). En el postoperatorio se constató tendencia a la hiponatremia al final del intervalo entre dosis consecutivas de Tolvaptán por lo que se ajustó la posología reduciendo la dosis (de 15 a $7,5 \mathrm{mg}$ ) y el intervalo interdosis (de 72 a 48 horas). En el seguimiento posterior se objetivó eunatremia mantenida estricta, con buena tolerancia al fármaco. Finalmente, en abril de 2015 , la paciente recibió un segundo curso de tratamiento QMT, sin que se evidenciaran alteraciones de la natremia durante el mismo (Tabla II).

\section{DISCUSIÓN}

El cáncer de ovario y las neoplasias ginecológicas en general son una etiología infrecuente de SIADH tumoral (4). En la literatura únicamente se han descrito 6 casos de SIADH secundario a tumores de ovario (3 teratomas inmaduros, 2 carcinomas y 1 cistoadenoma) $(5,6)$. El presenta caso representa, por tanto, el primero debido a un cistoadenocarcinoma.

Tabla I

ESTUDIO ANALÍTICO DE LA HIPONATREMIA

\begin{tabular}{lcc}
\hline Parámetro & Sangre (valores normales) & Orina \\
\hline Sodio & $126 \mathrm{mmol} / \mathrm{l}(135-145)$ & $113 \mathrm{mmol} / \mathrm{l}$ \\
Potasio & $3,9 \mathrm{mmol} / \mathrm{l}(3,5-5)$ & $69 \mathrm{mmol} / \mathrm{l}$ \\
Osmolaridad & $265 \mathrm{mOsm} / \mathrm{l}(275-295)$ & $725 \mathrm{mosm} / \mathrm{l}$ \\
\hline
\end{tabular}




\section{EVOLUCIÓN DE LA NATREMIA PARALELA AL TRATAMIENTO ONCOLÓGICO}

\begin{tabular}{|c|c|c|}
\hline Fecha & Tratamiento & $\begin{array}{l}\text { Sodio post-tratamiento } \\
(\mathrm{mmol} / \mathrm{l})\end{array}$ \\
\hline Junio 2013 & Cirugía radical & 130 \\
\hline Julio 2013 & Inicio QMT adyuvante & \\
\hline 10 Julio & $1^{\text {a }}$ sesión & - \\
\hline 31 Julio & $2^{\mathrm{a}}$ sesión & 127 \\
\hline 21 Agosto & $3^{\text {a }}$ sesión & 126 \\
\hline 11 Septiembre & $4^{\text {a }}$ sesión & - \\
\hline 2 Octubre & $5^{a}$ sesión & 134 \\
\hline 24 Octubre & $6^{a}$ sesión & 134 \\
\hline 15 Noviembre & $7^{a}$ sesión & 139 \\
\hline Octubre 2014 & Antes de Tolvaptán & 129 \\
\hline Diciembre 2014 & $\begin{array}{l}\text { Tolvaptán } \\
15 \mathrm{mg} / 72 \mathrm{~h}\end{array}$ & $138-143$ \\
\hline Enero 2015 & $\begin{array}{l}\text { Rescate quirúrgico + Tolvaptán } \\
7,5 \mathrm{mg} / 48 \mathrm{~h}\end{array}$ & $138-142$ \\
\hline Abril 2015 & $\begin{array}{l}\text { Segundo curso QMT + Tolvaptán } \\
7,5 \mathrm{mg} / 48 \mathrm{~h}\end{array}$ & $135-142$ \\
\hline
\end{tabular}

Las manifestaciones clínicas del SIADH son resultado de la hiperhidratación de las células neuronales, consecuencia, a su vez, de la hipoosmolaridad plasmática. Su intensidad puede ser leve, moderada o grave, en función de la severidad de la hiponatremia, y sobre todo de su velocidad de instauración. En hiponatremias crónicas (más de 48 horas de evolución) y de grado leve-moderado, como en nuestro caso, los síntomas son inespecíficos (anorexia, debilidad, náuseas) y a menudo atribuidos de forma errónea al propio tumor, por lo que el índice de sospecha debe ser elevado para identificar la verdadera causa de los mismos (7).

El diagnóstico de SIADH se establece por la presencia de hiponatremia hipoosmolar, osmolaridad en orina mayor de $100 \mathrm{mosm} / \mathrm{kg}$ y $\mathrm{Na}$ en orina mayor de 20-40 mmol/l, tras haber excluido hipotiroidismo, insuficiencia adrenal y uso de diuréticos. Una vez establecido el diagnóstico hay que buscar la causa subyacente. La producción ectópica de hormona antidiurética (ADH) por una neoplasia se considera la segunda causa más frecuente (aproximadamente el 30\% de los casos), siendo el cáncer microcítico de pulmón el causante de hasta el $80 \%$ de los casos de SIADH tumoral (8). En pacientes con cáncer es uno de los trastornos metabólicos más frecuentes (presente en 1-2\% de los oncológicos en general, y en el $10 \%$ de pacientes con cáncer microcítico de pulmón) (9). No obstante, en los pacientes oncológicos pueden coexistir otras causas de SIADH diferentes al propio tumor, lo que obliga a descartar otras etiologías (infecciones pulmonares, fármacos quimioterápicos del tipo vincristina, vinblastina, cisplatino o ciclofosfamida y alteraciones del sistema nervioso central).

Nuestra paciente recibió tratamiento con cisplatino, fármaco asociado con el desarrollo de SIADH (10). Sin embargo, la evolución de la natremia permite descartar su papel etiológico en este caso: la hiponatremia estaba ya presente antes del inicio de la QMT y mejoró con este tratamiento, paralelamente a la disminución de la carga tumoral

La medida terapéutica más importante en el SIADH crónico, consiste, siempre que sea posible, en eliminar la causa subyacente. En el caso del SIADH tumoral se trata de lograr el control eficaz de la neoplasia: típicamente la regresión tumoral se correlaciona con la resolución del SIADH, y la progresión tumoral con la recurrencia del trastorno $(11,12)$. De acuerdo con esta evolución, en nuestra paciente se observó una mejoría inicial de la natremia a partir de la quinta sesión de la QMT, coincidiendo con la reducción de la carga tumoral, y un empeoramiento posterior coincidiendo con la recidiva tumoral. Posteriormente, ante la imposibilidad de lograr la erradicación del tumor, fue necesario 
instaurar un tratamiento específico para controlar la hiponatremia (13). Las principales opciones terapéuticas en esta situación incluyen restricción hídrica, suplementos orales de sal, diuréticos de asa, demeclociclina, urea y vaptanes.

La restricción hídrica fue desestimada dado que el cálculo del índice de Furst ( $\mathrm{Na}+\mathrm{K}$ en orina/ $\mathrm{Na}$ en plasma) arrojó un resultado superior a 1 , prediciendo una nula respuesta a esta terapia. Los suplementos de sal y la urea oral se desestimaron por su mala tolerancia, y la demeclociclina por sus efectos tóxicos. El Tolvaptán es un antagonista selectivo del receptor de vasopresina V2. Al impedir la unión de la vasopresina a su receptor, y por tanto la movilización de la aquaporina 2 a la membrana celular, provoca un aumento de la acuaresis sin incrementar la pérdida de electrolitos (14). En nuestra paciente se consideró la opción terapéutica más adecuada pues se trata de un fármaco eficaz para la corrección de la hiponatremia leve-moderada por SIADH, con escasos efectos secundarios y disponible para administración oral (15). En el presente caso, el control de la hiponatremia se logró con dosis bajas del fármaco, confirmado la elevada sensibilidad de los pacientes con SIADH paraneoplásico al tratamiento con Tolvaptán, tal como se ha descrito previamente (16).

\section{CONCLUSIÓN}

El cáncer de ovario es una causa infrecuente de SIADH tumoral. La neoplasia suele estar diseminada cuando se presenta el SIADH, por lo que no es posible la curación de la neoplasia, y por tanto tampoco el control del SIADH, siendo necesaria la aplicación de otros tratamientos. El Tolvaptán ha demostrado ser eficaz para el control de la natremia, con una baja dosis, lo que confirma la elevada sensibilidad al fármaco que típicamente suelen presentar los SIADH paraneoplásicos.

\section{REFERENCIAS}

1. Ellison $\mathrm{DH}$, Berl T. The syndrome of inappropriate antidiuresis. N Engl J Med 2007;356(20):2064-72.

2. Goldvaser H, Rozen-Zvi B, Yerushalmi R, GafterGvili A, Lahav M, Shepshelovich D. Malignancy associated SIADH: Characterization and clinical implications. Acta Oncol 2016;55(9-10):1190-5.
3. Aylwin S, Burst V, Peri A, Runkle I, Thatcher N. 'Dos and don'ts' in the management of hyponatremia. Curr Med Res Opin. 2015;31(9):1755-61.

4. Martina C. Murphy and Merry J. Markham. Rare case of syndrome of inappropriate antidiuretic hormone as the initial presentation of ovarian cancer recurrence. $J$ Clin Oncol 2015;33(9):e48-9.

5. Roman H, Verspyck E, Sentilhes L, Barau G.. Immature ovarian teratoma with hyponatremia and low antidiuretic hormone level. Obstet Gynecol 2004;103(5 Pt 2):1108-10.

6. Lam SK, Cheung LP. Inappropriate ADH secretion due to immature ovarian teratoma. Aust N Z J Obstet Gynaecol 1996;36(1):104-5.

7. van Meerbeeck JP, Fennell DA, De Ruyssche DK. Small-cell lung cancer. Lancet 2011;378(9804):174155.

8. Murphy MC, Markham MJ. Rare case of syndrome of inappropriate antidiuretic hormone as the initial presentation of ovarian cancer recurrence. J Clin Oncol 2015;33(9):e48-9.

9. Yokoyama $\mathrm{Y}$, Shigeto $\mathrm{T}$, Futagami M, Mizunuma $\mathrm{H}$. Syndrome of inappropriate secretion of anti-diuretic hormone following carboplatin-paclitaxel administration in a patient with recurrent ovarian cancer. Eur $\mathrm{J}$ Gynaecol Oncol 2005;26(5):531-2.

10. Vanhees SL, Paridaens R, Vansteenkiste JF. Syndrome of inappropriate antidiuretic hormone associated with chemotherapy-induced tumor lysis in small-cell lung cancer: case report and literature review. Ann Oncol 2000;11(8):1061-5.

11. Pelosof LC, Gerber DE. Paraneoplastic syndromes: an approach to diagnosis and treatment. Mayo Clin Proc. 2010;85(9):838-54.

12. Castillo JJ, Vincent M, Justice E. Diagnosis and management of hyponatremia in cancer patients. Oncologist 2012;17(6):756-65.

13. Runkle I, Villabona C, Navarro A, Pose A, Formiga F, Tejedor A, Poch E. [The treatment of hyponatremia secundary to the syndrome of inappropriate antidiuretic hormone secretion]. Med Clin (Barc) 2013;141(11):507.e1-507.e10.

14. Schrier RW, Gross $P$, Gheorghiade M, Berl T, Verbalis JG, Czerwiec FS, Orlandi C; SALT Investigators. Tolvaptan, a selective oral vasopressin V2-receptor antagonist, for hyponatremia. N Engl J Med 2006; 355(20):2099-112.

15. Cuesta M, Thompson CJ. The syndrome of inappropriate antidiuresis (SIAD). Best Pract Res Clin Endocrinol Metab 2016;30(2):175-87.

16. Kenz S, Haas CS, Werth SC, Bohnet S, Brabant G. High sensitivity to tolvaptan in paraneoplasic syndrome of inappropriate ADH secretion (SIADH). Ann Oncol 2011;22(12):2696. 